\title{
Article \\ A Case Study on User Evaluation of Scientific Publication Summarization by Japanese Students
}

\author{
Shintaro Yamamoto ${ }^{1,2, *}$, Ryota Suzuki $^{2}{ }^{2}$, Tsukasa Fukusato ${ }^{3}$, Hirokatsu Kataoka ${ }^{2}\left(\mathbb{C}\right.$ and Shigeo Morishima ${ }^{4}$ \\ 1 Graduate School of Advanced Science and Engineering, Waseda University, Tokyo 169-8050, Japan \\ 2 National Institute of Advanced Industrial Science and Technology (AIST), Tsukuba 305-8560, Japan; \\ ryota.suzuki@aist.go.jp (R.S.); hirokatsu.kataoka@aist.go.jp (H.K.) \\ 3 Graduate School of Information Science and Technology, The University of Tokyo, Tokyo 113-8654, Japan; \\ tsukasafukusato@is.s.u-tokyo.ac.jp \\ 4 Waseda Research Institute for Science and Engineering, Tokyo 169-8050, Japan; shigeo@waseda.jp \\ * Correspondence: s.yamamoto@fuji.waseda.jp
}

Citation: Yamamoto, S.; Suzuki, R.; Fukusato, T.; Kataoka, H.; Morishima, S. A Case Study on User Evaluation of Scientific Publication Summarization by Japanese Students. Appl. Sci. 2021, 11, 6287. https://doi.org/10.3390/ app11146287

\section{Academic Editors: Valentina}

Franzoni, Alfredo Milani and Giulio Biondi

Received: 10 June 2021

Accepted: 4 July 2021

Published: 7 July 2021

Publisher's Note: MDPI stays neutral with regard to jurisdictional claims in published maps and institutional affiliations.

Copyright: (c) 2021 by the authors. Licensee MDPI, Basel, Switzerland. This article is an open access article distributed under the terms and conditions of the Creative Commons Attribution (CC BY) license (https:// creativecommons.org/licenses/by/ $4.0 /)$.

\begin{abstract}
Summaries of scientific publications enable readers to gain an overview of a large number of studies, but users' preferences have not yet been explored. In this paper, we conduct two user studies (i.e., short- and long-term studies) where Japanese university students read summaries of English research articles that were either manually written or automatically generated using text summarization and/or machine translation. In the short-term experiment, subjects compared and evaluated the two types of summaries of the same article. We analyze the characteristics in the generated summaries that readers regard as important, such as content richness and simplicity. The experimental results show that subjects are mainly judged based on four criteria, including content richness, simplicity, fluency, and format. In the long-term experiment, subjects read 50 summaries and answered whether they would like to read the original papers after reading the summaries. We discuss the characteristics in the summaries that readers tend to use to determine whether to read the papers, such as topic, methods, and results. The comments from subjects indicate that specific components of scientific publications, including research topics and methods, are important to judge whether to read or not. Our study provides insights to enhance the effectiveness of automatic summarization of scientific publications.
\end{abstract}

Keywords: user evaluation; scientific publication; academic activity analysis; language processing application

\section{Introduction}

With the increasing number of scientific publications [1], scientific researchers (including university students with little research experience) are often faced with an enormous number of studies in their domains of interest. It can be very time-consuming and tedious to read these papers. To alleviate this burden, it is important to find ways whereby papers can be surveyed more efficiently. In this paper, we focus on a summary which briefly describes an overview of scientific research.

One straightforward approach is to read only the "abstract" sections (which are manually written by authors) of papers. While abstracts are intended to attract readers [2], Elkiss et al. mentioned that abstracts can also be used as summaries of scientific publications [3]. In general, the abstracts of foreground published papers have been evaluated during the peer-review process by third party researchers, so their quality is guaranteed. However, several researchers claimed that abstracts used do not necessarily meet readers' requirements because they are written from the perspective of authors [3,4]. Hence, instead of author-written abstracts, it is important to stand in the place of the reader who may not have substantive domain-relevant knowledge and construct brief descriptions that help users gain an overview of research papers. In this paper, we consider 
two types of summaries: abstracts written by authors and text that briefly describes an entire paper's overview. There are two ways to generate summaries from an original paper. Several research groups in Japan have attempted to manually construct summaries of academic articles to share knowledge concerning computer science research in, for example, the field of Human-Computer Interaction (HCI) [5] and Computer Vision (CV) [6]. As the manual summaries are written from the perspective of readers, it is possible to help other readers understand the research without having to read the target papers. In addition, these manual summaries are written in Japanese, so they are advantageous for these non-native English speakers. However, writing summaries manually is still difficult and tedious since the first readers (who write summaries) must understand the target research papers to a significant extent. In addition, these summaries have not been reviewed by others (i.e., professional reviewers) and the quality of summaries (e.g., adequacy of information) is not guaranteed.

To alleviate the issue of manual summary writing, automatic generation of research summaries (written in readers' mother tongues) from the original papers has been demanded. In the field of Natural Language Processing (NLP), automatic summarization [7] and machine translation [8] are active research topics. Although these techniques can generate research summaries without any manual effort, the majority of work on text summarization has focused on news articles, which are markedly different from scientific publications in terms of text length or document structure $[4,9,10]$. Moreover, research on manual evaluation of automatic summarization also utilizes news articles as material [11]. Investigating users' preference and request is beneficial since summaries are intended to convey information to humans, but the majority of automatic summarization research relies on automatic evaluation metrics [12]. To establish a framework to automatically generate summaries of scientific papers, we need to elicit readers' opinions about research article summaries as a first step.

In this work, we conduct a case study wherein Japanese university students read summaries written manually (including abstract by authors or summary by readers) and generated automatically (using NLP techniques) to investigate what characteristics readers require in terms of summaries of scientific papers. Specifically, we performed two experiments (Figure 1): (1) comparing two summaries generated with different methods to investigate the characteristics in summaries that readers regard as important, and (2) reading a large number of summaries to investigate what characteristics readers use to determine whether to read original papers after reading the summaries. In experiment 1 , we investigate what factors of summaries are important through comparison. Though several factors, including content and grammatical correctness, are assumed, we clarify what is important to users. In experiment 2, we assume the practical scenario that users read summaries to find relevant papers. The purpose here is to investigate which kind of information is helpful to judge whether users are interested in papers. Our sample is framed in terms of university students because these subjects are unlikely to have substantive prior research experience and expert knowledge in their field and therefore need to survey a lot of scientific literature. 


\section{Experiment 1}

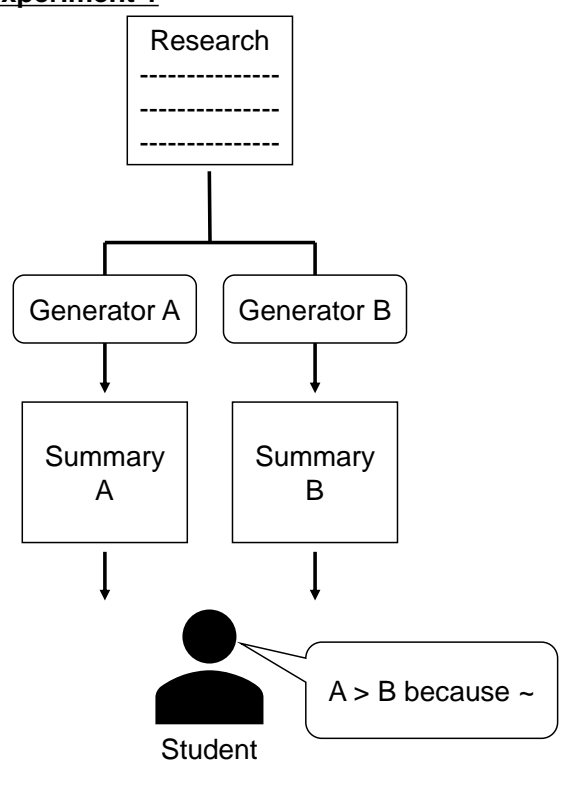

\section{Experiment 2}

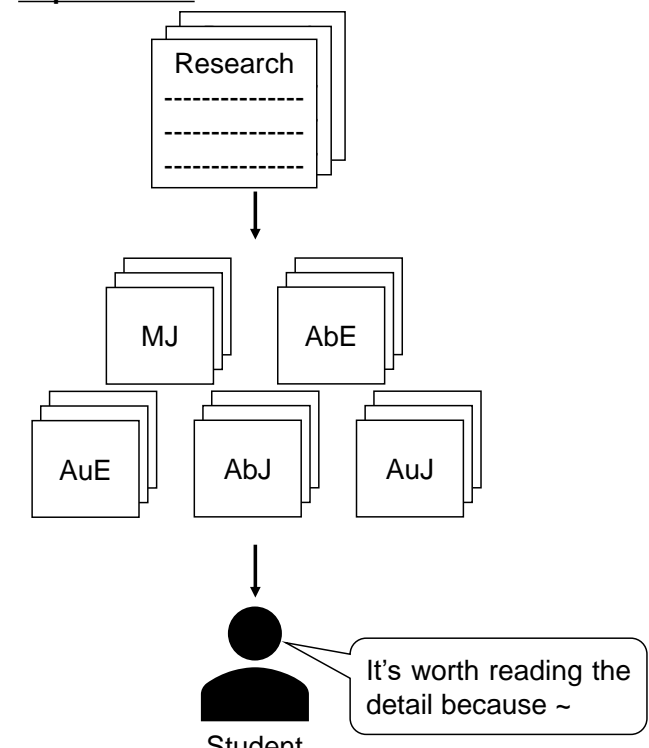

Student

Figure 1. Experimental configuration. In experiment 1, subjects compare two types of paper summaries generated with different methods, named "summary A" and "summary B." In experiment 2, subjects read summaries and judge whether they would like to read the corresponding papers within four weeks.

\section{Related Work}

In the NLP field, an automatic generation of text summaries is an active topic [7] and several researchers have focused on scientific publications $[4,9,10,13-16]$. The majority of research on text summarization has focused on news articles $[4,9,10]$. However, summarizing scientific publications raises unique issues compared to summarizing news articles. For example, scientific papers tend to be longer than news articles (often much longer) and scientific articles follow normalized discourse structures including background, experimental settings, and results [17]. Accordingly, research on scientific publication summarization relies on particular characteristics, for example, section-based [9], structure-based [4,10,14], and citation-based methods $[4,10,13]$.

Lloret et al. [18] point out that text summarization results should be evaluated manually based on readability and the appropriateness of the information they contain. In [11], systems to generate summaries are evaluated by human readers using four criteria: relevance, consistency, fluency, and coherence. The authors of [11] also compared human responses with automatic evaluation metrics used in text summarization research [12]. Huang et al. [19] defined eight errors including missing key points or unnecessary repetition and asked users to manually select errors in computer generated summaries to investigate the limitations of prevailing automatic summarization methods. In these studies, the CNN/DM dataset [20], which consists of news articles, is used for manual evaluation of summaries. Compared with news articles, an important characteristic in scientific publication is that target readers are limited to researchers with prior knowledge, as well as text-length and article structure. In this work, inspired by these studies, we conducted a user study of reading summaries of scientific papers.

In general, university students working on Bachelor's or Master's theses do not have enough experience of research activities, so we think that summaries are needed to help students understand the main point of research papers. Ozuru et al. [21] investigated the effect of prior knowledge and reading skill on scientific text comprehension. Their results indicate that comprehension is positively correlated with prior knowledge. Shepherd and Van De Sande [22] observed that reading strategies of faculty members in mathematics are different from those of undergraduate students. In addition, August et al. [23] studied 
science communications and revealed that specialized terminology is often used, which creates access barriers for beginners. Hence, we focus on university students as subjects, and investigate the characteristics in summaries that they regard as important.

Scientific research papers are generally written in English, but it is preferred that readers can read in their mother tongues. This is because non-native English speakers tend to get lower scores on science examinations conducted in English, compared with native English speakers [24]. Previously, Tobin and McRobbie [25] investigated science education for Chinese students in an English-speaking school, specifically an Australian high school. They found that those students prefer to have conversations in Chinese and take notes in Chinese. In this paper, if we conduct a user study with non-native English speakers (Japanese), we need to investigate the influence of language differences. We consider two major directions to obtain information using subjects' mother tongues: manual translation and automatic translation. In terms of the latter, machine translation systems have been well studied and freely accessible online systems like Google translate [26] are available. Regarding manual translation, Japanese research groups have attempted to manually construct Japanese summaries in some research fields [5,6]. However, manual translations are time-consuming. Therefore, we utilized both automatic translation and existing manual summaries constructed by Japanese research groups to investigate differences between the two approaches.

\section{Problem Formulation}

We conducted user studies on reading summaries of scientific publication. The main purpose is to investigate the strengths and weaknesses of manual and automatic summaries, and readers' expectations concerning scientific paper summarization. In addition, we assume that translating summaries into readers' mother tongues influences their understanding. Then, we also investigated the effect of scientific article summaries written in English and readers' mother tongues (in this paper, Japanese).

\subsection{Subjects}

The studies were conducted with undergraduate and graduate students working on their own research. This is because many students do not have prior research experience, but they have to investigate prior research to grasp trends and unresolved problems in their own field. Lack of research experience results in difficulty of reading a scientific paper, for example, students face unfamiliar terminology. In addition, motivations of research activities are different among students (e.g., pursuing research activities by enrolling in a $\mathrm{Ph} . \mathrm{D}$. course, or graduating from their university only), and there are different levels of abilities for understanding overviews of scientific papers according to each individual or university course, for example, language skill and basic knowledge. To sum up, we think that undergraduate and graduate students are good subjects for our user studies because they have specific problems of research activities (e.g., prior knowledge) and subjects' variance are also expected.

We invited native Japanese students (including 1st and 2nd year Master's students and 4th year Bachelor's students) who are working in the CV field or related areas (e.g., Computer Graphics) as listed in Table 1. Note that some of them participated in either experiment 1 or 2 due to their schedule. We considered students from three universities in Japan to ensure diversity. A Univ. and B Univ. are private universities, and C Univ. is a national university. Based on these universities' official information, admission to A Univ. is more competitive than B Univ. and the difficulty of admission to C Univ. is between those of A Univ. and B Univ., and students in A Univ. are more likely to pursue doctoral studies. In the case of A Univ. and B Univ. subjects, their laboratories both consist of more than 20 students, while laboratory at C Univ. consists of less than 10 students. 
Table 1. Subjects, their characteristics, and experiments in which they participated.

\begin{tabular}{lcccc}
\hline Subject & University & Degree (Year) & English Skill & Experiment \\
\hline ABH01 & A Univ. & Bachelor's (4) & High a & Experiment 1, 2 \\
ABM02 & A Univ. & Bachelor's (4) & Medium & Experiment 1 \\
ABM03 & A Univ. & Bachelor's (4) & Medium & Experiment 1, 2 \\
ABM04 & A Univ. & Bachelor's (4) & Medium & Experiment 1, 2 \\
AmM05 & A Univ. & Master's (1) & Medium & Experiment 1, 2 \\
BBL06 & B Univ. & Bachelor's (4) & Low & Experiment 1, 2 \\
BBL07 & B Univ. & Bachelor's(4) & Low & Experiment 1,2 \\
BBL08 & B Univ. & Bachelor's (4) & Low & Experiment 2 \\
BmM09 & B Univ. & Master's (1) & Medium & Experiment 1,2 \\
BmM10 & B Univ. & Master's (1) & Medium & Experiment 1,2 \\
BmL11 & B Univ. & Master's (1) & Low & Experiment 1 \\
BmL12 & B Univ. & Master's (1) & Low & Experiment 1, 2 \\
BML13 & B Univ. & Master's (2) & Low & Experiment 1,2 \\
BML14 & B Univ. & Master's (2) & Low & Experiment 1 \\
CmM15 & C Univ. & Master's (1) & Medium & Experiment 1,2 \\
CmM16 & C Univ. & Master's (1) & Medium & Experiment 1,2 \\
CMM17 & C Univ. & Master's (2) & Medium & Experiment 1,2 \\
CMM18 & C Univ. & Master's (2) & Medium & Experiment 1, 2 \\
\hline
\end{tabular}

a This subject had lived in the U.S. for 10 years.

Reading a text written in English is one of the barriers for non-native students including Japanese. To understand the differences in reading comprehension of non-native students with different English skill, we classified the subjects based on their English skill (low, middle, and high). We referred to the score of TOEIC Listening \& Reading Test (https:/ / www.iibc-global.org/english/toeic/test/lr.html last accessed on 9 June 2021) (minimum and maximum scores are 10 and 990, respectively) because TOEIC is a well-known English test in Japan, and most Japanese universities often require taking a TOEIC examination many times to students. According to the average score of Japanese (https:/ / www.iibc-global.org/library/default/english/toeic/official_data/pdf/ Worldwide2019_SW_english.pdf last accessed on 9 June 2021), we consider that subjects whose score is lower than 500 are low-level and subjects whose score is between 500 and 700 are medium-level. Here, we denote subjects with the initial characters of university, grade, English skill, and subject number. For example, the subject 01, who is a student of A Univ. in a Bachelor course and has High English skill, is denoted as ABH01. Regarding the grade of Master's students, 1st and 2nd year's are expressed as $\mathrm{m}$ and M, respectively. The subject ABH01 had lived in the U.S. for 10 years and her/his score in TOEIC is much higher than the others. Moreover, only ABH01 mentioned after the experiments that s/he had no difficulty in English during the experiments. To distinguish this participant from the others, we regard ABH01 as high-level. There are few students with living experience in foreign countries and having special interests in CV field, and, therefore, we can obtain relevant data from those subjects who have no difficulty with both the English and Japanese languages.

\subsection{Reading Material}

As reading material, we randomly selected 70 papers ( 20 and 50 for the short-term experiment and the long-term experiment, respectively) which were accepted to the IEEE/CVF Conference on Computer Vision and Pattern Recognition (CVPR) 2018. This conference is one of the most successful conferences in the field of computer vision (CV) (https: / / cvpr2018.thecvf.com/ last accessed on 9 June 2021).

Over 3300 papers were submitted to CVPR 2018 and 979 of them were accepted (i.e., the acceptance rate is about $29.7 \%$ ), indicating the difficulty of getting accepted to CVPR. 
Next, we prepared five-different summaries (see Table 2): (1) Manual Japanese (MJ): Japanese summaries written as part of a Japanese research project [6]. To manually construct all of the accepted paper summaries in the CV field, this project took around three months for each $\mathrm{CV}$ conference. Note that this project has no requirement for summary length as long as it is no longer than a single page. For our experiments, we chose summaries written by three professional Japanese researchers who received a Ph.D. degree in either the $\mathrm{CV}$ field or a related domain within the past five years. The average number of Japanese characters per summary is $294.9 \pm 48.6$. (2) AbE (Abstract English): We use English abstracts written in the original papers by the authors. The average number of words per summary is $164.3 \pm 30.2$. (3) AuE (Automatic English): English summaries generated by an automatic text summarization method. To achieve the assistance of reading comprehension, it is necessary to select an appropriate summarization method without dependency on any additional data, which is applicable to any scientific research paper. We employed an unsupervised algorithm, called TextRank [27], to compute sentence importance based on sentence connectivity and generate summaries that consist of important sentences only. TextRank is still a strong baseline which shows competitive performance to a recent summarization method [19]. Note that we exclude the "abstract" and the "reference" sections in the original papers and use only the paper bodies as inputs. To compute importance, we use variations of the similarity function [28] implemented in the gensim library (https: / / radimrehurek.com/gensim/ last accessed on 9 June 2021).

In addition, we empirically set the maximum number of words in each summary to 250 words. This is because Cohan and Goharian [10] reported that each summary contains 235.6 words on average in a benchmark for scientific publication summarization TAC 2014 (http:/ / tac.nist.gov/2014/BiomedSumm/ last accessed on 9 June 2021). (4) AbJ (Abstract Japanese): We translate AbE into Japanese. We employ Google translate (https: / / translate.google.com last accessed on 9 June 2021), which is a well-known and freely available machine translation system. (5) AuJ (Automatic Japanese): Japanese summaries translated from AuE (English) into Japanese. As with the AbJ preparation, we also use Google translate.

Table 2. Types of summaries used in our experiments.

\begin{tabular}{clcc}
\hline & Type & Summarization Method & Language \\
\hline $\mathrm{MJ}$ & (Manual Japanese) & Manually written by researchers with Ph.D. & Japanese (written by native speakers) \\
$\mathrm{AbE}$ & (Abstract English) & Papers' abstract & English \\
$\mathrm{AuE}$ & (Automatic English) & Automatic summarization & English \\
$\mathrm{AbJ}$ & (Abstract Japanese) & Papers' abstract & Japanese (Google translate) \\
$\mathrm{AuJ}$ & (Automatic Japanese) & Automatic summarization & Japanese (Google translate) \\
\hline
\end{tabular}

\subsection{Experimental Setting}

As an experiment setting, we made two experimental plans in the case of Japanese students summary reading. In experiment 1 , we asked the subjects to compare the above types of summaries. Several factors can contribute to a user's evaluation on summaries like content and grammatical correctness. We assume that the subjects compare summaries based on better or worse aspects of summaries. We then asked the subjects to provide the reason for their judgement, which could be considered as the description of strength or weakness of summaries. From the comments provided by the subjects, we investigated the evaluation factors and the characteristics of each summary.

In experiment 2, we asked the subjects to read 50 summaries (10 of each type) and to answer whether they would like to read the original papers after reading their summaries only within four weeks. Summaries of scientific publications can be used to find relevant research without reading the entire text of an article. We consider the task in experiment 2 as finding papers' interest from a collection of (randomly selected) 50 papers. We investigated important characteristics to determine which papers to read under the practical scenario of 
finding relevant papers. Compared with experiment 1 , here, the conditions are meant to emulate a real use case for summaries, for example, wherein the subjects need to conduct a task afterward (e.g., writing code).

\section{Experiment 1: Paired Comparison}

In experiment 1, we prepared pairs of summaries as mentioned in Section 3 (see Table 2). Each subject was asked to evaluate all possible combinations (10 pairs, in total), and to describe the reasons for this evaluation, so that we could understand the evaluation criteria.

\subsection{Experimental Setting \\ 4.1.1. Subjects}

We asked 17 native Japanese students to participate in experiment 1 as shown in Table 1 . Note that the number of subjects satisfies the experimental standard in the field of HCI [29].

\subsubsection{Experimental Methodology}

We randomly selected 20 papers in CVPR 2018 and prepared summaries. The subjects had not read the original papers. The subjects were asked to compare pairs of summaries from a paper ("summary A" vs. "summary B"). For each pair, we randomly selected one different original paper, and subjects were asked to evaluate all possible combinations (i.e., 10 pairs). We asked the subjects "which summary is better at providing an understandable overview of the papers, summary A or summary B?" using the score metric in Table 3. Subjects were also asked to "describe reasons for the evaluation" in addition to the score metric. Note that the instructions to subjects and subjects' answers are in Japanese. The purpose of the questions is to highlight the strengths and weaknesses of each summary thorough comparison, and, therefore, we provided minimum instructions not to lead the answer. We explained that both "summary A" and "summary B" were constructed based on the same paper, but the subjects were not informed of the summary types. The order of the combinations (for example, the first pair is "MJ and AbE," and the next is "AbE and $\mathrm{AuJ}$ ") was randomly shuffled. In addition, we did not set a time limit for reading each pair. To reduce the bias caused by the order and times of reading, the subjects were allowed to read either A or B first and to reread summaries if they wished. Note that the accuracy of readers' understanding is beyond the scope of this study. The evaluation process took approximately 90 min per subject.

Table 3. Evaluation metrics in experiment 1.

\begin{tabular}{cl}
\hline Score & \multicolumn{1}{c}{ Meaning } \\
\hline+2 & A is better than B. \\
+1 & A is slightly better than B. \\
0 & A and B are of the same quality. \\
-1 & B is slightly better than A. \\
-2 & B is better than A. \\
\hline
\end{tabular}

\subsection{Experimental Results}

\subsubsection{Analysis Method}

We denote the score of pairwise summaries (A,B) by a subject $n$ as $s_{n}(A, B)\left(=-s_{n}(B, A)\right)$. For each type of summary $m \in\{M J, A b E, A u E, A b J, A u J\}$, we normalize the score as follows:

$$
\alpha_{m}=\frac{1}{N M} \sum_{n=1}^{N} \sum_{m^{\prime} \neq m}^{M} s_{n}\left(m, m^{\prime}\right)
$$


where $\alpha_{m}$ indicates the score of summary type $m$, and $N$ and $M$ are the number of subjects and summary types, respectively (in this paper, $N=17$ and $M=5$ ). We use Scheffe's method [30] (modified method of Nakaya [31]) to verify the existence of difference among score distributions. If a significant difference exists $(p<0.05)$, we can obtain the pair $(\mathrm{A}, \mathrm{B})$ which is significantly different by calculating a yardstick value.

The experiment was conducted in Japanese except for summaries written in English $(\mathrm{AbE}$ and $\mathrm{AuE})$. All answers in Japanese were translated into English by the authors.

\subsubsection{Score}

The mean and standard deviation of relative score for pair $(\mathrm{A}, \mathrm{B})$, and normalized score $\alpha_{m}$ are summarized in Tables 4 and 5, respectively. From the yardstick at $p<0.05$ $Y_{0.05}=1.04$, we find significant differences in $(\mathrm{AbE}, \mathrm{AuJ})$ and $(\mathrm{AbE}, \mathrm{AuE})$ (see Table 4, bold fonts).

Table 4. Means and standard deviations of scores in experiment 1 for each pair. Higher mean scores indicate that $\mathrm{A}$ is better than $\mathrm{B}$. Emboldening indicates significant difference.

\begin{tabular}{cccccc}
\hline & & \multicolumn{4}{c}{ B } \\
\cline { 3 - 6 } & & AbE & AuE & AbJ & AuJ \\
\hline \multirow{4}{*}{$\mathrm{A}$} & MJ & $0.06 \pm 1.48$ & $0.76 \pm 0.90$ & $-0.24 \pm 1.52$ & $1.18 \pm 1.02$ \\
& $\mathrm{AbE}$ & - & $\mathbf{1 . 3 5} \pm \mathbf{1 . 0 6}$ & $0.47 \pm 1.46$ & $\mathbf{1 . 4 7} \pm \mathbf{0 . 5 1}$ \\
& $\mathrm{AuE}$ & - & - & $-0.41 \pm 1.46$ & $0.06 \pm 1.30$ \\
& $\mathrm{AbJ}$ & - & - & - & $0.65 \pm 1.37$ \\
\hline
\end{tabular}

Table 5. Summary scores according to subjects' attribute. The higher the number, the better the summary.

\begin{tabular}{ccccccccccc}
\hline \multirow{2}{*}{ Type } & \multirow{2}{*}{ All } & \multicolumn{3}{c}{ University } & \multicolumn{3}{c}{ Degree (Year) } & \multicolumn{3}{c}{ English Skill } \\
& & A Univ. & B Univ. & C Univ. & Bachelor's (4) & Master's (1) & Master's (2) & Low & Medium & High \\
\hline $\mathrm{MJ}$ & 0.35 & 0.32 & 1.00 & -0.12 & 0.43 & 0.43 & 0.10 & 0.57 & 0.26 & 0.00 \\
$\mathrm{AbE}$ & 0.65 & 0.60 & 0.63 & 0.75 & 0.83 & 0.20 & 1.15 & 0.87 & 0.48 & 1.00 \\
$\mathrm{AuE}$ & -0.49 & -0.36 & -0.96 & -0.64 & -0.37 & -0.49 & -0.70 & -0.53 & -0.56 & -0.60 \\
$\mathrm{AbJ}$ & 0.16 & 0.16 & -0.12 & 0.52 & -0.20 & 0.37 & 0.35 & -0.20 & 0.34 & 0.60 \\
$\mathrm{AuJ}$ & -0.67 & -0.72 & -0.92 & -0.64 & -0.70 & -0.51 & -0.90 & -0.70 & -0.62 & -1.00 \\
\hline \#subjects & 17 & 5 & 8 & 4 & 6 & 7 & 4 & 6 & 10 & 1 \\
\hline
\end{tabular}

According to these results, it is thought that the abstracts written by the original authors of the papers are better than the summaries generated by the NLP approach. This indicates that the automatic text summarization used in this work does not satisfy the user's requirement. On the other hand, for example, the mean score of (MJ,AbE) is 0.06 , which indicates that $\mathrm{MJ}$ is slightly better, but there is no significant difference between $\mathrm{MJ}$ and AbE. Eight subjects answered that "MJ is (slightly) better than AbE," whilst nine stated that "AbE is (slightly) better than MJ." That is, opinions toward the pair (MJ,AbE) are divided among subjects.

\subsubsection{Comments from Subjects}

The evaluation results show that abstracts from the original paper (AbE) achieve the best score and manual summaries written in Japanese (MJ) are second (see Table 5). Here, we analyze subjects' comments and reveal the evaluation factors.

Subjects' comments can be classified into one or more of the following four types; (1) content richness, (2) simplicity, (3) fluency, and (4) format (see Table 6). In terms of content richness, subjects noted whether the main contents of scientific papers (e.g., research purpose and experimental results) were included, and summaries with such information tended to get a higher score. In the case of simplicity, subjects answered whether summaries 
were simple to read or not. It was considered that simple summaries were preferred; however, they should not be too simple. Indeed, about $20 \%$ of the comments on simplicity stated that "summaries are too simple to grasp the overall meaning of the research." Moving on to fluency, this evaluation metric was concerned with whether subjects can read summaries without language difficulties, for example, whether a summary is written in their mother tongue or whether it is grammatically correct. In our experiment, some subjects mentioned that "summaries written in Japanese are easy to understand". By contrast, other subjects also answered that "English is better than incorrect Japanese" and "I'm familiar with terminology in English." In the case of format, subjects evaluate based on the appearance of texts. For example, if summaries referred to figures or tables, the subjects keep lower scores because the output summaries consisted only of texts. Other comments that cannot be classified into the above four types include "I cannot understand either summary" and "there is no difference between the two summaries." None of the comments that fall into the other types mention any characteristics of the summaries.

Table 6. Users' comments in experiment 1.

\begin{tabular}{|c|c|c|c|}
\hline Type of $R$ & son & \#Answers & Example \\
\hline \multirow{2}{*}{ Content richness } & Strength & 41 & $\begin{array}{l}\text { (In the former summary,) problem and purpose are clear and easy } \\
\text { to understand. (by CMM18) }\end{array}$ \\
\hline & Weakness & 12 & $\begin{array}{l}\text { (In the latter summary,) I could not understand the purpose of } \\
\text { research. (by BBL07) }\end{array}$ \\
\hline \multirow{2}{*}{ Simplicity } & Strength & 49 & $\begin{array}{l}\text { (The latter summary) describes the important points briefly and } \\
\text { thus I could get an overview easily. (by CmM15) }\end{array}$ \\
\hline & Weakness & 25 & $\begin{array}{l}\text { (The latter summary is) too simple so I did not think it was good. } \\
\text { (by CMM18) }\end{array}$ \\
\hline \multirow{2}{*}{ Fluency } & Strength & 36 & $\begin{array}{l}\text { The content is the same, so the summary in Japanese is preferred. } \\
\text { (by ABM03) }\end{array}$ \\
\hline & Weakness & 56 & (In the former summary,) the Japanese was strange. (by ABM04) \\
\hline \multirow[b]{2}{*}{ Format } & Strength & 0 & No answer is given \\
\hline & Weakness & 32 & $\begin{array}{l}\text { Though the summary refers to a figure and a table, I could not } \\
\text { access them. (by AmM05) }\end{array}$ \\
\hline Others & & 6 & There is no difference between the two summaries. (by AmM05) \\
\hline
\end{tabular}

\subsubsection{Attributes of Subjects}

We also analyzed the results in terms of subjects' attributes (i.e., university, degree and English skill). Per attribute score of each summary and distribution of reason are shown in Table 5 and Figure 2, respectively.

Those subjects who were 4th year Bachelor's students and those with low English skill are more likely to mention weakness in fluency. The same tendency can be observed in B Univ., but this is because all subjects with low English skill are students at B Univ. Around $85 \%$ of the comments concerning weakness in fluency mention the linguistic error caused by machine translation. AbJ (Japanese abstract) received a lower score by subjects with low English skill and 4th-year Bachelor's students (Table 5). Thus, we can infer that subjects with these attributes tend to care more about linguistic errors by machine translation.

Similarly, three out of ten comments by ABH01, who has experience of living in the U.S. for 10 years, are about linguistic errors in Japanese from machine translation. This subject has better English than all others and did not mention "Japanese is preferred." For the above reasons, summaries created without machine translation (i.e., MJ and AbE) tend to be preferred in terms of grammatical and word errors. 
Regarding the difference in university, students at A Univ. tend to mention strengths in simplicity as well as English in B Univ. From the data obtained in this experiment, the reasons for the differences in summary requests with regard to the subjects' universities are unclear. One possible explanation is that each university or research group has policies which may influence student preferences.

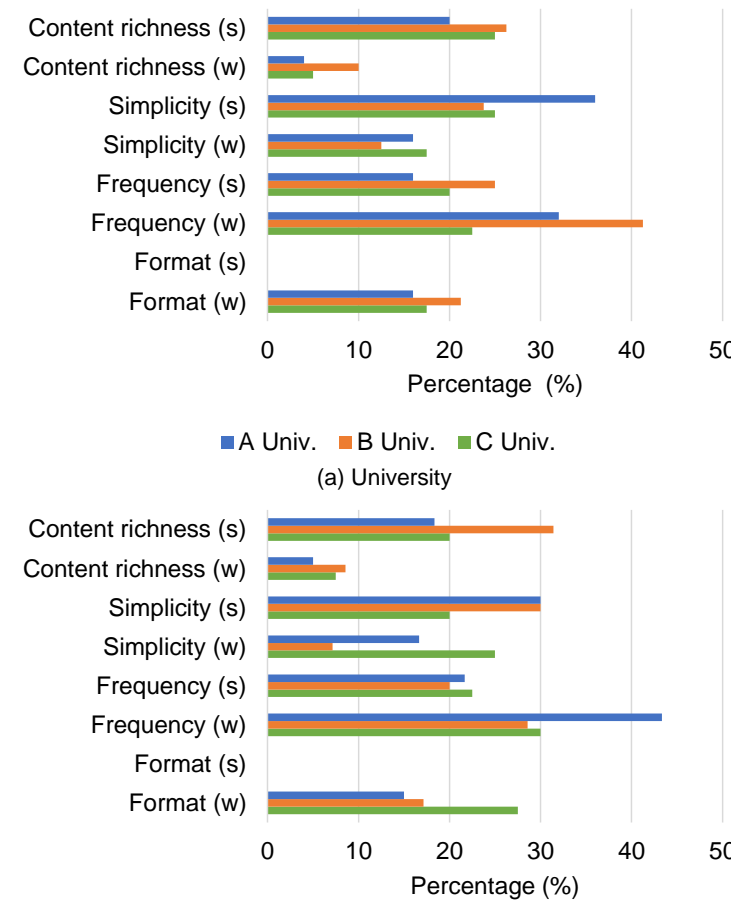

घachelor's (4) $\quad$ Master's (1) Master's (2)

(b) Degree (year)

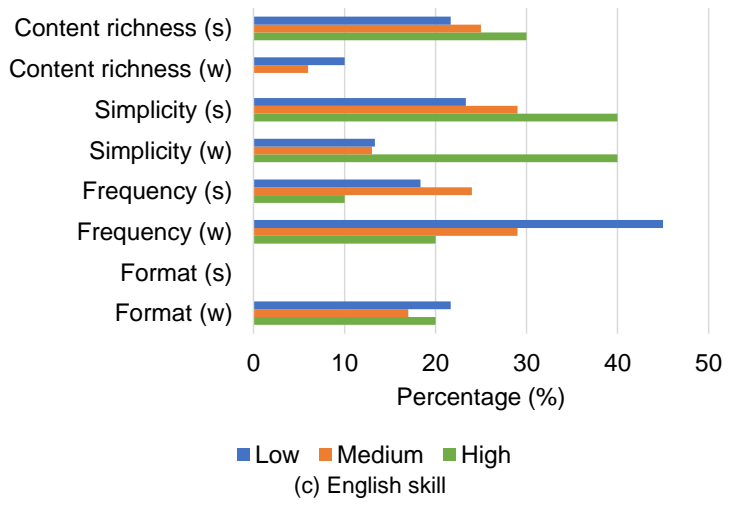

Figure 2. Distributions of the reasons for subjects' answers for their (a) university, (b) degree year, and (c) English skill, in experiment 1 . Note that " $\mathrm{s}$ " and " $\mathrm{w}$ " indicate strength and weakness, respectively.

\section{Experiment 2: Reading Thorough}

As previously stated, summaries of scientific papers are useful for readers to decide whether to read the original paper. For example, Semantic Scholar (https: / / www. semanticscholar.org/ last accessed on 9 June 2021), an academic search engine, shows single-sentence paper summaries generated by an automatic summarization method [32] to help users judge which papers are relevant. Besides this, a Japanese HCI research group [5] created a web-based system which enables manual recording interesting papers by clicking a button after reading summaries, named "read this paper later". Although such systems are suitable for sharing interesting summaries and papers, they do not consider how to determine whether a paper is interesting. Hence, we performed the second 
experiment to investigate what characteristics in summaries readers may use to decide whether to read the original paper after reading summaries.

\subsection{Experimental Setting}

\subsubsection{Subjects}

We invited the same 14 students who formed the sample in experiment 1 , plus one who did not participate in experiment 1 (15 students, in total). All subjects are Japanese students working in the CV field or related domains. Please see Table 1 for the subjects' details.

\subsubsection{Experimental Methodology}

We randomly selected 50 papers accepted to CVPR 2018 and divided them into five groups (10 papers each). We then applied one of the methods (Table 2) to each group and 50 summaries were generated in total.

We developed a web-based system (see Figure 3) to manage unread summaries. We constructed two functions: (1) a user-account management function to gauge subjects' progress (for instructors), and (2) a display function to visualize the number of papers which each subject read (for subjects). Subjects created an account to manage their individual progress.

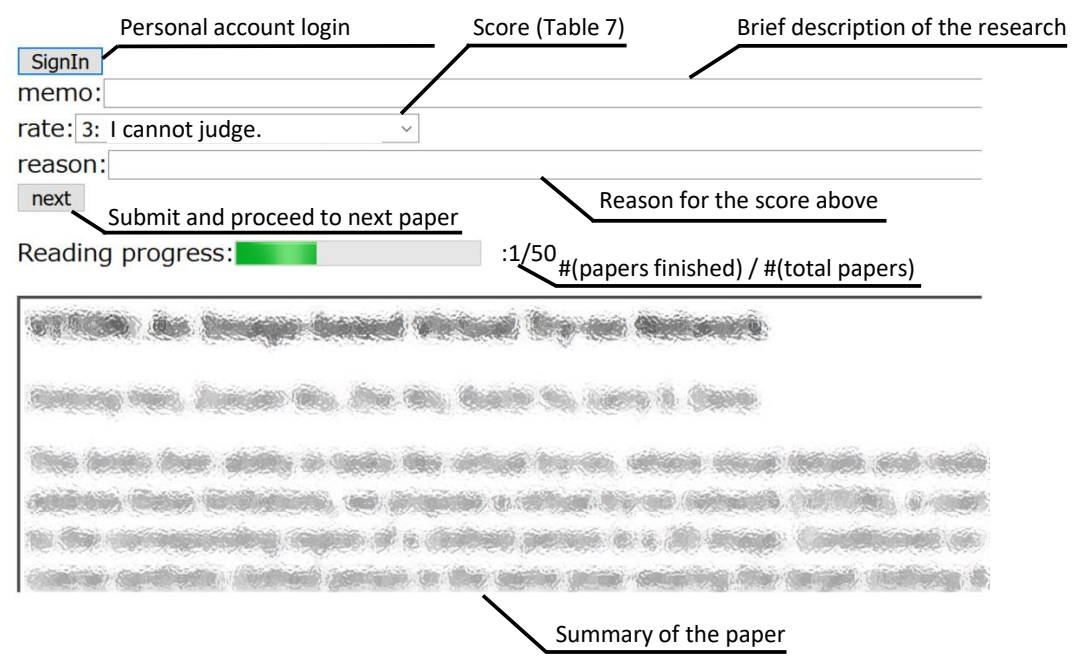

Figure 3. Our web-based system that subjects used in experiment 2. The system displays 50 summaries one by one in the bottom panel. After reading each summary, the user answers whether s/he would like to read the corresponding paper by using five-point Likert scores (see Table 7) and its reasons (text).

For each paper, subjects were asked to read a summary displayed on our web system and to answer whether they would like to read the original paper. We asked the subjects to answer the following three questions regarding the summary that they read on the system: "brief description of the research they could understand from the summary," "score of whether they would like to read the original paper to understand the papers' detail after reading the summary (using five-point Likert scale in Table 7)," and "what is of interest to subjects." The instructions and subjects' answers are in Japanese. We then clarified what factor of summaries is useful to find relevant papers from the answers. Subjects were asked to finish the steps above for all 50 summaries within four weeks. Note that the system randomly shuffled the reading order of 50 summaries according to the subject accounts.

In this experiment, we assume the real scenario that the subjects have several tasks like coding and they read summaries in between other tasks. We then instructed that the subjects could read the summaries at their own pace (e.g., reading all summaries at once or a few per day) as long as they could give their answers within four weeks. We sent an e-mail each week to remind subjects of the deadline. In the case that subjects could 
not finish reading all 50 summaries, we collected only the answers which were submitted. After experiment 2, we also asked the subjects to give their opinions about the summaries that they had read.

Table 7. Evaluation metric in experiment 2.

\begin{tabular}{cl}
\hline Score & \multicolumn{1}{c}{ Criteria } \\
\hline 5 & I would strongly like to read it. \\
4 & I would like to read. \\
3 & I cannot judge. \\
2 & I would not like to read. \\
1 & I would strongly not like to read. \\
\hline
\end{tabular}

\subsection{Experimental Results}

\subsubsection{Score}

Within the experimental period (four weeks), 11 out of 15 subjects finished answering all of the prepared summaries. The remaining four subjects finished answering 30, 19, 15, and 11 summaries, respectively. The scores are summarized in Table 8.

We ran a Mann-Whitney's U test (two-tailed hypothesis) to evaluate the difference in the responses with our 5-point Likert scale (Table 8). The U-values indicate that the following pairs are significant at $p<0.05$ : (MJ, AbE), (MJ,AuE), (MJ, AbJ), (MJ, AuJ), $(\mathrm{AbE}, \mathrm{AuE}),(\mathrm{AbE}, \mathrm{AbJ})$, and $(\mathrm{AbE}, \mathrm{AuJ})$. Moreover, we computed the Pearson's correlation coefficient between the scores in experiment 1 (see Table 5) and experiment 2 (see Table 8 , respectively), and the coefficient was $r=0.80$. Therefore, the scores representing whether to read (i.e., the result in experiment 2) was highly correlated with the score for the pairwise comparison in experiment 1.

Table 8. Summary scores according to subjects' attributes in experiment 2.

\begin{tabular}{|c|c|c|c|c|c|c|c|c|c|c|}
\hline \multirow{2}{*}{ Type } & \multirow{2}{*}{ All } & \multicolumn{3}{|c|}{ University } & \multicolumn{3}{|c|}{ Degree (Year) } & \multicolumn{3}{|c|}{ English Skill } \\
\hline & & A Univ. & B Univ. & C Univ. & Bachelor's (4) & Master's (1) & Master's (2) & Low & Medium & High \\
\hline Overall & 3.30 & 3.08 & 3.26 & 3.54 & 3.31 & 3.18 & 3.60 & 3.33 & 3.34 & 2.84 \\
\hline MJ & 3.93 & 3.91 & 3.85 & 4.19 & 3.98 & 3.97 & 3.40 & 3.57 & 3.96 & 4.18 \\
\hline $\mathrm{AbE}$ & 3.59 & 3.72 & 3.34 & 4.00 & 3.75 & 3.19 & 4.38 & 3.53 & 3.56 & 4.00 \\
\hline $\mathrm{AuE}$ & 3.02 & 2.92 & 2.99 & 3.19 & 2.92 & 3.04 & 3.25 & 3.02 & 3.15 & 2.20 \\
\hline $\mathrm{AbJ}$ & 3.07 & 2.62 & 3.11 & 3.34 & 3.08 & 3.04 & 3.12 & 3.18 & 3.13 & 2.00 \\
\hline $\mathrm{AuJ}$ & 2.87 & 2.62 & 2.91 & 3.00 & 2.89 & 2.78 & 3.06 & 2.89 & 2.92 & 2.40 \\
\hline \#subjects & 15 & 4 & 7 & 4 & 6 & 6 & 3 & 5 & 9 & 1 \\
\hline
\end{tabular}

\subsubsection{Comments from Subjects}

Subjects' comments in experiment 2 can be divided into two categories, as shown in Table 9. The first type is about "interest." A lot of subjects mentioned that they scored based on their interests; for example, the topic was close to their research topic, and they would like to know more detailed experimental results. The second category is based on "quality or comprehensiveness." While some subjects mentioned that they could not understand the summary due to its quality, others mentioned that their interest was stimulated because the summary was high quality. In the case that the comment mentioned both "interest" and "quality or comprehensiveness," we counted both. Note that three responses were not considered as one of these two types, in which subjects mentioned that they had already read the paper before. 
Table 9. Scoring reasons in experiment 2.

\begin{tabular}{|c|c|c|c|c|c|c|}
\hline \multicolumn{7}{|c|}{ (a) The count of reason of each type. } \\
\hline & \multirow{2}{*}{ Type of Reason } & \multicolumn{5}{|c|}{ \#answers } \\
\hline & & MJ & $\mathrm{AbE}$ & AuE & $\mathrm{AbJ}$ & $\mathrm{AuJ}$ \\
\hline \multirow{2}{*}{ Interest } & Positive & 83 & 70 & 56 & 50 & 48 \\
\hline & Negative & 22 & 36 & 21 & 40 & 30 \\
\hline Quality or comprehension & Positive & 28 & 19 & 7 & 13 & 8 \\
\hline Others & & 1 & 0 & 2 & 0 & 0 \\
\hline \multicolumn{2}{|c|}{ Mean score } & $3.93 \pm 0.84$ & $3.59 \pm 0.93$ & $3.02 \pm 1.18$ & $3.07 \pm 1.09$ & $2.87 \pm 1.20$ \\
\hline \multicolumn{2}{|c|}{ Total answers } & 129 & 121 & 126 & 122 & 127 \\
\hline \multicolumn{7}{|c|}{ (b) The examples of the reason. } \\
\hline \multicolumn{2}{|r|}{ Type of reason } & \multicolumn{5}{|c|}{ Example } \\
\hline Interest & Positive & \multicolumn{5}{|c|}{$\begin{array}{l}\text { The topic is relevant to my research. (by BBL07) } \\
\text { The topic is not relevant to my research. (by AmM05) }\end{array}$} \\
\hline \multirow{2}{*}{ Quality or comprehension } & Positive & \multirow{2}{*}{\multicolumn{5}{|c|}{$\begin{array}{l}\text { I became interested in the research because the summary was high quality. (by BML13) } \\
\text { I could not understand the summary. (by BmM10) }\end{array}$}} \\
\hline & Negative & & & & & \\
\hline Others & \multicolumn{6}{|c|}{ I have read the paper before. (by ABH01) } \\
\hline
\end{tabular}


We discuss the comments about "interest" in more detail. The answers about "interest" can be classified according to the components of scientific papers (e.g., topic, method, and experiment), as shown in Table 10. We consider that comments mentioning multiple components are counted for each component, and 14 answers were of this type. In the case of the topic component, the subjects scored whether they had an interest in the research topic or relevance with their own research topic. In the case of the methods' component, the subjects mentioned that they would like to know the algorithm or technique. Additionally, some mentioned that they would like to know the detailed experimental results. Here, we consider comments that did not mention any concrete component as "other".

Table 10. Distribution of interest-related comments regarding the research content.

\begin{tabular}{lcl}
\hline & \#Comments & \multicolumn{1}{c}{ Example } \\
\hline Topic & 280 & I am interested in face recognition. (by CmM16) \\
Method & 78 & I am interested in the algorithm. (by BBL08) \\
Experiment & 18 & I would like to know the synthesis result. (by ABM04) \\
Other & 84 & I am interested. (by BBL06) \\
\hline
\end{tabular}

\subsubsection{Subsequent Question}

After experiment 2, we also asked the subjects to answer whether the four characteristics in experiment 1 (see Table 6) are important or not. The responses from the subjects are summarized in Figure 4.
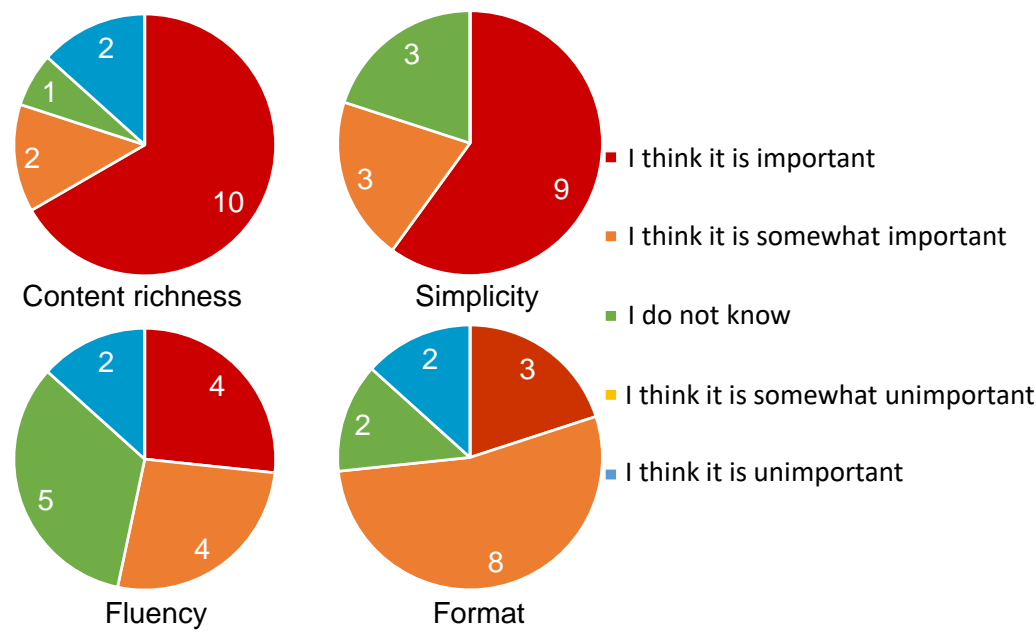

Figure 4. Subjects' opinions about the importance of the four evaluation factors from experiment 1.

For the question about the importance of content richness, about $80 \%$ of the subjects answered either "I think it is important" or "I think it is somewhat important." On the other hand, two subjects answered "I do not know" or "I think it is somewhat unimportant". Their reasons were that "the detailed experimental results are not necessary" (by AmM05), and "content richness is not necessary and I would rather know a little bit each about a larger number of papers" (by CMM17).

In the case of simplicity, around $80 \%$ of subjects agreed that simplicity is important. The reasons include "a longer summary is difficult to understand" (by CmM16) and "I would like to quickly read an easily understandable summary" (by CmM15). On the other hand, three subjects answered "I do not know" because "I cannot understand the summary with insufficient information even if it is simple" (by ABH01, BmL12, and BML13). From the answers for content richness and simplicity, it is considered that these aspects need to be well balanced. 
In case of fluency, $53.4 \%$ of subjects placed importance on fluency, which is lower than the percentages for the importance of content richness and simplicity. To investigate the importance of fluency further, we also asked subjects to answer "how often did you prefer Japanese summaries, even if the text was grammatically incorrect, compared to English ones ( $0 \%$ : English is always better, $100 \%$ : Japanese is always better)" to investigate the effect of the mother tongue (see Table 11). All subjects with low-level English skills answered more than " $50 \%$," and one subject with medium-level English skill answered " $80 \%$." ABH01, who had lived in the U.S. for 10 years, answered "0\%." The reasons are including "appropriateness is more important than whether the summary is written in the mother tongue or not" (answer: 20\%, by ABM03), "I usually read scientific papers in English" (answer: 10\%, by CMM17) and "I can read text in the mother tongue quickly" (answer: 70\%, by CmM16).

Table 11. Preference for mother tongue even with incorrect language. In addition, $50 \%$ indicates that mother tongue is preferred in $50 \%$ of cases, even when grammatically incorrect.

\begin{tabular}{cccccccccccc}
\hline Percentage(\%) & $\mathbf{0}$ & $\mathbf{1 0}$ & $\mathbf{2 0}$ & $\mathbf{3 0}$ & $\mathbf{4 0}$ & $\mathbf{5 0}$ & $\mathbf{6 0}$ & $\mathbf{7 0}$ & $\mathbf{8 0}$ & $\mathbf{9 0}$ & $\mathbf{1 0 0}$ \\
\hline \# answers & 1 & 2 & 1 & 0 & 2 & 1 & 4 & 2 & 2 & 0 & 0 \\
\hline
\end{tabular}

For the importance of format, $73.3 \%$ of subjects answered "I think it is important" or "I think it is somewhat important." Examples of reasons are "incorrect formatting bothers me every time" (by ABM04) and "I cannot understand the text because of inappropriate formatting" (by CMM18). On the other hand, those who answered "I think it is somewhat unimportant" pointed out that "I can correct the text in my mind" (by ABM03) or "even if the format is incorrect, it does not make hard to read" (by BmM09). While some did not care about incorrect format, most did.

\subsubsection{Attribute of Subjects}

As with experiment 1 (see Section 4), we also analyzed the results per attribute (e.g., grade of university and English skill). Per attribute score of each summary and distributions of reason are shown in Table 8 and Figure 5, respectively.

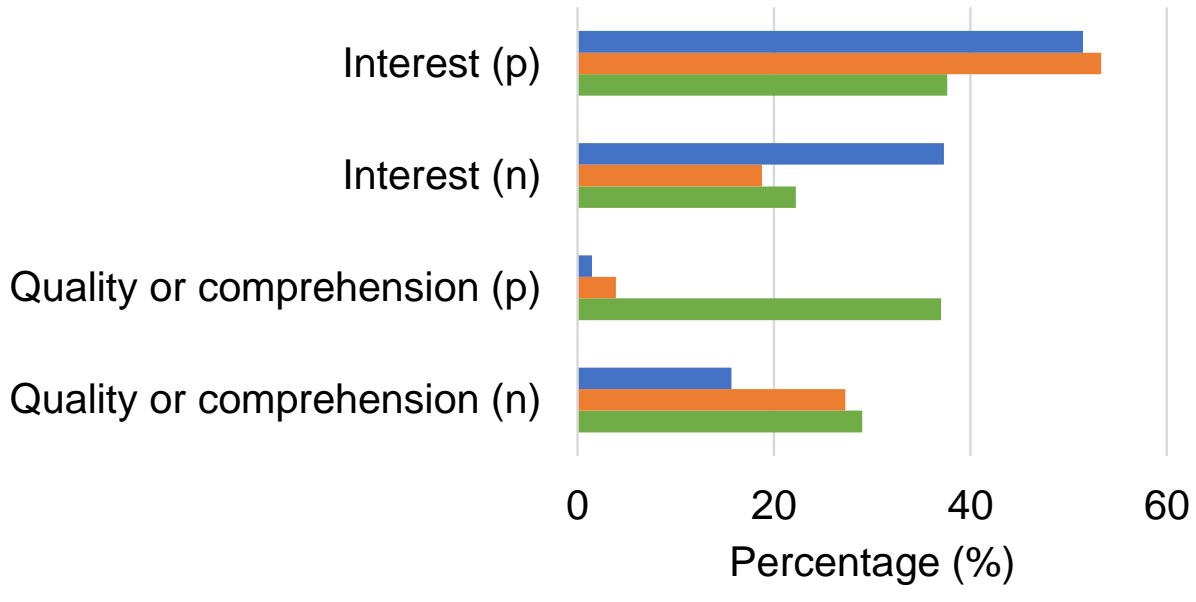

$\square$ A Univ. $\quad$ B Univ. $\quad$ C Univ.

(a) University

Figure 5. Cont. 


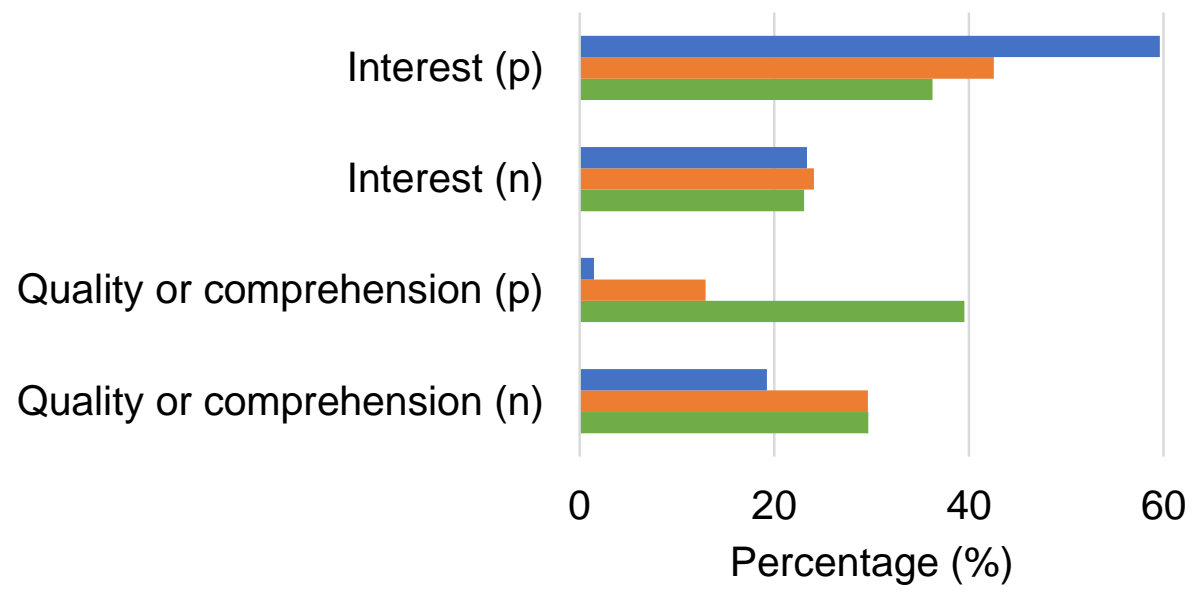

-Bachelor's (4) - Master's (1) \# Master's (2)

(b) Degree (year)

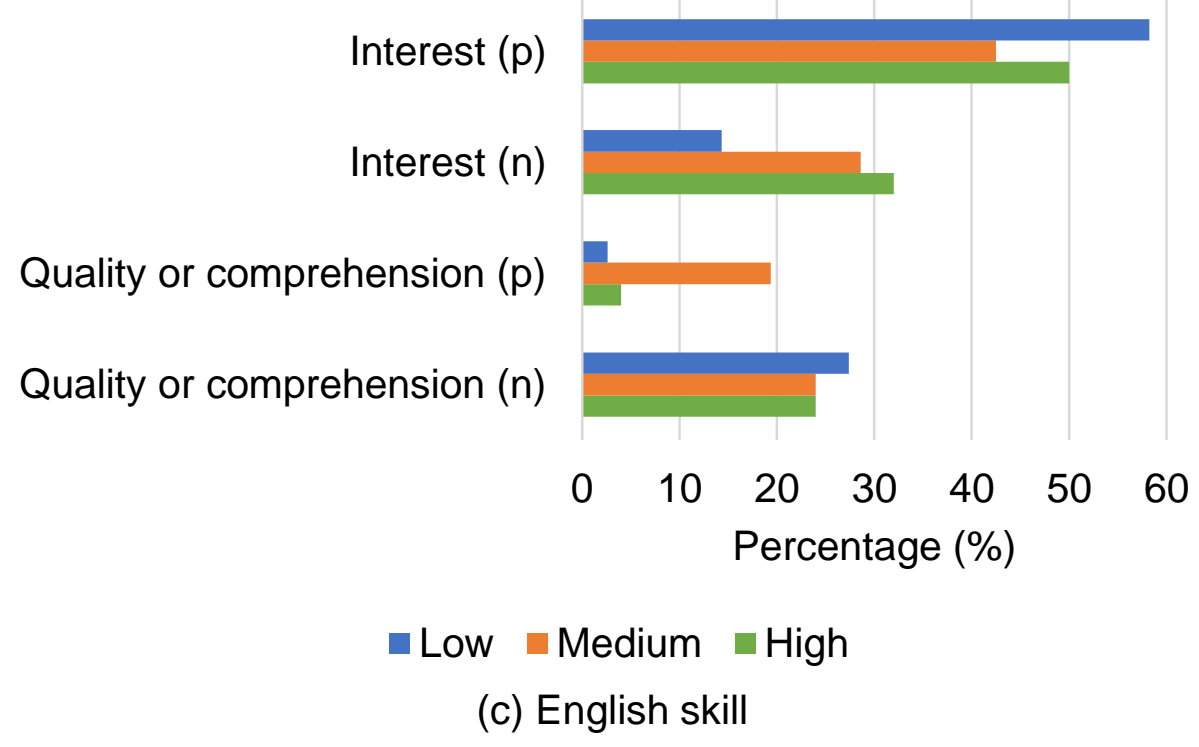

Figure 5. Type of reasons underlying subjects' answers (including positive and negative comments) disaggregated by characteristics: (a) university; (b) degree year; (c) English skill, in experiment 2. Note that " $\mathrm{p}$ " and " $\mathrm{n}$ " indicate positive and negative, respectively.

The results indicate that upper-grade students tended to show less positive interest. On the other hand, upper-grade students tended to mention more positive aspects of quality or comprehension. One possible reason is that undergraduate students have less prior knowledge and training, which results in focusing on finding relevant research, while seniors are more familiar with the background of their field and focus more on the quality of comprehension of the summaries.

Regarding the university, the subjects at $C$ Univ. mentioned more positive aspects of quality or comprehensiveness. On the other hand, they also tended to give answers indicating less positive interest.

\section{Discussion}

In this section, based on the two experiments (see Sections 4 and 5), we further discuss (1) students' evaluations and requests regarding summaries of scientific publications, and (2) characteristics of manual and automatic generated summaries in English and Japanese. We also describe some concerns of this study for future directions. 


\subsection{Evaluation Metric and Request}

In experiment 1 , subjects evaluated summaries based on content richness, simplicity, fluency, and format. Fluency and format, which can be regarded as general aspects of summarization, are relevant in terms of readability as pointed out by Lloret et al. [18]. On the other hand, we find specific aspects of scientific papers in content richness and simplicity summarized as follows:

- Subjects (university students) tend to evaluate qualities of summaries based on the existence of components of scientific papers such as research purpose, methods, and experimental results. To understand overviews of papers, we observe that they did not use all components.

- Subjects tended to prefer simple summaries, but additional information is also required such as an explanation of terminology.

\subsubsection{Paper Components and Content Richness}

Concerning content richness, as shown in Table 6, the subjects commented on whether there are important components of scientific papers (e.g., topic and method) in the summaries. For example, some subjects' answers are about papers' components, like, for example, "the problem of prior work and the purpose of research are easy to understand" (by AmM05) and "the purpose and the proposed method are not clear" (by CmM16). In general, scientific publications have a common structure consisting of multiple sections, including a background, experimental setup, and results [17], so most readers (students) will briefly evaluate and confirm whether these structures appear in the summary of a paper. However, such components in summaries have not been studied in previous text summarization research because the focus therein has tended to be on "news" articles, which do not have any common structure. In addition, to evaluate the appropriateness of the information in a summary, Nenkova and Passonneau [33] proposed a pyramid method to place importance on the information mentioned in more reference summaries, but this method does not consider any structural components such as those in scientific papers.

In experiment 2, the subjects recorded whether they would like to read the original papers after reading their summaries. We observed that subjects mainly made this judgment based on either "interest" or "quality and comprehension." In the answers about "interest," $62.6 \%$ and $17.4 \%$ were about research topic and method, respectively, so it appears that subjects tend to regard these components as most important. Note that $3 \%$ of the answers mentioned multiple components of research articles. This indicates that subjects paid attention to a limited number of components. Cohan and Goharian $[4,10]$ proposed summarization methods to equally select some sentences from each component (e.g., methods and results) based on the structure of scientific publication. While these systems balance the amount of each component, it can be said that they do not consider the importance of each component.

Moreover, the Master's students tend to have less positive attitudes in terms of interest. One possible reason is that upper-grade students have already obtained specific knowledge about their research fields, which could result in more attention being paid to the quality or comprehension of summaries. Thus, the importance of each component depends on readers' attributes (e.g., background of research topic). From the above, we can infer that considering readers' preferences for components are important to improve the qualities of scientific research paper summarizations.

\subsubsection{Simplicity and Terminology}

Regarding simplicity, subjects mentioned that "a simple summary is preferred" and also that "the summary is too simple to understand," as shown in Table 6. About $80 \%$ of subjects gave answers indicating that they preferred simple summaries, for example, "this summary is too long to read" (by BBL07) and "this summary gives an overview simply and is easy to understand" (by ABM04). In addition, in answering the related question in experiment 2 , about $80 \%$ of subjects also expressed a preference for simple summaries. 
Simplifying document sequences is an active research topic in the NLP field, and two types of simplification methods have been studied: lexical and syntactic [34]. One characteristic of scientific research papers is the existence of terminology. Authors assume that readers have prior knowledge of the field, so they often leave out explanations of terminology. As a result, university students without prior knowledge, especially Bachelor's and Master's students, must deal with unfamiliar vocabulary. Indeed, some subjects mentioned, for example, "I could not understand both summaries. I am not familiar with the terminology so I could not understand it" (by ABH01) and "supplementing the terminology is helpful" (by BmL12). These comments are consistent with research on science communication [23], where specialized terminology has been shown to create access barriers for new users. For this reason, although redundant sentences are not desired, a lexical simplification or explanations of the terminology are required.

\subsubsection{Automatic Evaluation Metric}

Manual evaluation of summaries is very labor-intensive. Thus, automatic evaluation metrics have been well used in automatic summarization research. Here, we discuss the effectiveness of automatic evaluation metrics in scientific paper summarization.

ROUGE [12] is a well-known evaluation metric, which measures similarity between generated and reference summaries. We applied ROUGE to the summaries in our experiment with the following settings (see Table 12): (1) AuE with AbE as reference, (2) AbJ and $\mathrm{AuJ}$ with $\mathrm{MJ}$ as reference, and (3) $\mathrm{AuJ}$ with $\mathrm{AbJ}$ as reference. As a result, $\mathrm{AuJ}$ obtains higher ROUGE score with AbJ as reference than MJ. However, in the comparison between MJ and $\mathrm{AbJ}$ (see experiment 1 ), about $40 \%$ of subjects answered " $\mathrm{AbJ}$ is better," which was not a significant difference. ROUGE measures the similarity of a generated summary to a reference summary, so the metric does not indicate whether the quality of a generated summary is high," but "the generated summary is similar to the reference." Therefore, until we can guarantee the quality of reference summaries, we cannot use ROUGE. In addition, content richness and simplicity are difficult to evaluate from the similarity with reference summaries. This discussion is consistent with the experimental results in Kryscinski et al. [11], where it was reported that manual evaluations such as appropriateness or fluency are not correlated with ROUGE scores.

Table 12. ROUGE scores of summaries used in experiment 1.

\begin{tabular}{ccccc}
\hline Type & ROUGE-1 & ROUGE-2 & ROUGE-L & Reference \\
\hline $\mathrm{AuE}$ & 20.70 & 5.71 & 10.81 & $\mathrm{AbE}$ \\
$\mathrm{AbJ}$ & 36.09 & 7.54 & 19.40 & $\mathrm{MJ}$ \\
$\mathrm{AuJ}$ & 30.62 & 6.27 & 16.13 & $\mathrm{MJ}$ \\
$\mathrm{AuJ}$ & 49.30 & 16.18 & 22.56 & $\mathrm{AbJ}$ \\
\hline
\end{tabular}

\subsection{Characteristics of Manual and Automatic Summarization}

We evaluated five types of summaries generated by manual or automatic summarization methods (in addition to a machine translation system). We found the following characteristics of each type summary from the experimental results:

- Manual Japanese was easy to read compared with automatic summarization because of the terminology explanation as well as fluency. However, there were some negative opinions as well, such as "it is too simple" and "there is insufficient information on summaries."

- Automatic summarization methods obtained negative feedback, for example, "there is too much unnecessary information." On the other hand, subjects also answered "there is important information (in automatically generated summary) that is not included in another one (manual summary)" when they compared automatically and manually generated summaries. There is the possibility of reducing or removing the perceptual biases of the authors. 
- Machine translation systems may make grammatical errors and word errors due to the existence of specialized terminology that is not used in other domains (e.g., news articles), but some subjects preferred summaries written in Japanese, even if grammatically incorrect, over English ones.

\subsubsection{Manual Summarization}

We prepared (a) English abstracts in the original papers (AbE) and (b) manual Japanese summaries written by researchers with a Ph.D. (MJ). In experiment 1 (see Table 5), AbE obtained the highest score of 0.65 and MJ obtained the second highest score 0.35 , indicating that manual summarization is highly evaluated. Note that the MJ score was not far behind, since there is not any significant difference with $\mathrm{AbE}$.

However, in the case of $\mathrm{AbE}$, some subjects answered " $(\mathrm{MJ}$ is better than $\mathrm{AbE}$ because MJ) describes meaningful information about the test accuracy with all datasets in the papers' experiments" (by AmM05) and "(AuE is better than AbE because AuE) describes what is done in this research, while the other one (AbE) does not" (by BmM10). According to these comments, we can see that $\mathrm{AbE}$ does not always include important information for readers. This point is consistent with the claim by several researchers that abstract does not always reflect readers' requirements [3,4].

On the other hand, MJ summaries depend on the Japanese researchers' subjective judgment, but it is also possible to include important information (which is not written in the AbE summary) for increased ease of understanding by considering the readers' perspective. We also observed comments about document simplification in MJ, for example "(MJ) seems to be re-written by humans who have read the original paper carefully, so I feel (MJ) is easy to understand" (by BmM10) and "(MJ) is good because it describes the meaning of the terminology" (by BmL12). However, some subjects also mentioned low fluency like "the Japanese is incorrect in (MJ) and there is typographical error" (by ABM04). There was also a comment that MJ is too simple to understand: "(MJ) is not bad but too simple" (by CMM17). One of the possible reasons for the above negative comments is that $\mathrm{MJ}$ is not checked by third-party readers, while the abstract $(\mathrm{AbE})$ is evaluated during peer review. In addition, MJ is written by researchers who have obtained a Ph.D., but the subjects in our experiments are undergraduate and Master's students. Therefore, the gap in prior knowledge might be a reason for the aforementioned comments that MJ summaries are "too simple."

In comparison between $\mathrm{MJ}$ and $\mathrm{AbE}$ in the experiment 1, eight subjects answered "(MJ) is better" and nine answered "(AbE) is better," which indicates that opinions are divided in half. Among those who answered "(MJ) is better," five subjects mentioned the reason is that MJ is written in Japanese. Four subjects, including one who mentioned that Japanese is preferred, pointed out terms of simplicity or content richness. According to these comments, we can see that $\mathrm{AbE}$ is not necessarily better in simplicity and content richness.

Whereas we have observed several characteristics of manual summarization through the experiments, it is not easy to draw any definite conclusions regarding the evaluation of manual summarization. This inconclusiveness indicates that more data are necessary while writing a summary of scientific papers is time-consuming. Additionally, the difficulty of concluding is also a sign that evaluation criteria could be more explicit as summaries are evaluated in a subjective manner in this study.

\subsubsection{Automatic Summarization}

Automatic summarization methods were used to prepare $\mathrm{AuE}$ and $\mathrm{AuJ}$. In experiment 1 , these summaries obtained lower scores and significant differences in the (AbE,AuJ) and $(\mathrm{AbE}, \mathrm{AuE})$ pairs were found, as shown in Table 5. One of the reasons why these summaries obtained lower scores is the existence of information that was deemed useless. Subjects gave comments such as "I cannot understand what (AuE) describes at all. There are too many unnecessary information" (by BML14). In addition, some subjects gave answers concerning consistency, for example, "(AuE) begins with 'our contributions mainly 
lie in three aspects,' but only one of the aspects is mentioned" (by AmM05). According to these results, it is thought that one limitation of existing summarization methods (in this paper, we used TextRank [27]) as the lack of consistency as pointed out in [35].

On the other hand, we can see that AuE tackles one problem of abstracts, namely that information required by readers is not necessarily included [4]. For example, some subjects answered that $\mathrm{AuE}$ is better than $\mathrm{AbE}$ or $\mathrm{AbJ}$. Comments given included "( $\mathrm{AuE}$ is better than $\mathrm{AbE}$ because $\mathrm{AuE}$ ) describes what is done in this research paper, while the other one $(\mathrm{AbE})$ does not" (by BmM10) and "(AuE) first clarifies a final goal with the phrase 'the main objective of this paper.' In addition, using the phrase 'towards this goal,' it is easy to understand the actual results" (by BML13). From these comments, it is considered that, by using automatic summarization methods, it may be possible to reduce issues regarding the original abstracts $(\mathrm{AbE})$ being biased due to the author's point of view, which is one of the advantages of these methods.

In addition, the automatic summarization method used in our experiments extracts important sentences from a target paper without any modification, so serious grammatical errors and word errors should not occur with these methods. This assumption is also supported by answers from one subject with high-level English skills (ABH01), who commented, "I think that grammatical errors and word errors were found only in Japanese summaries."

\subsubsection{Machine Translation System}

We translated $\mathrm{AbE}$ and $\mathrm{AuE}$ into Japanese using Google translate as $\mathrm{AbJ}$ and $\mathrm{AuJ}$, respectively. The results of experiment 1 show that translated summaries (AbJ and $\mathrm{AuJ}$ ) obtained lower scores than the original ones ( $\mathrm{AbE}$ and $\mathrm{AuE}$ ), as shown in Table 5. However, there were no any significant differences for the $(\mathrm{AbE}, \mathrm{AbJ})$ and $(\mathrm{AuE}, \mathrm{AuJ})$ pairs.

Negative feedback for machine translation is mostly about grammatical errors and word errors due to terminology translation and consistency. For example, some subjects mentioned that "semantic segmentation is difficult to translate in Japanese" (by BML13) and "( $\mathrm{AuJ}$ ) is difficult too read, even if the summary is written in Japanese" (by BmM10). One possible reason is that the translation of specific words such as "proposed method" and "prior work" are wrong because the Google translate system is not intended for research articles.

On the other hand, $35 \%$ of the subjects answered that they preferred translated summaries ( $\mathrm{AbJ}$ and $\mathrm{AuJ})$ in experiment 1 . In addition, in subsequent questions of experiment 2 , about $60 \%$ of subjects answered "I think that $50 \%$ of the Japanese summaries, even if they contain grammatical errors, are better." Therefore, although there are grammatical errors and word errors in the machine translated results, some subjects may feel that there is an advantage in reading summaries in their mother tongue (Japanese).

\subsection{Limitation Concerns for Future Directions}

Here, we discuss some concerns of this study that can be tackled in future work. This study utilized automatic text summarization and machine translation techniques to generate a Japanese summary from a scientific paper written in English. The evaluation by the subjects could be affected by the qualities of the two methods, but it does not remain easy to distinguish these factors. For future work, it will be important to handle two factors separately. One possible solution is to prepare manually written summaries in English as well as in Japanese. Preparing manually written summaries in English and Japanese enables a comparison of manually generated summaries in Japanese written by a native Japanese speaker and translated from English into Japanese. It is also possible to compare manually and automatically generated summaries in English, which was not considered in this study.

Furthermore, in our experiments, we did not separate the benefit of translation into Japanese and linguistic error caused by machine translation. To investigate the advantage of translation into Japanese, evaluating summaries translated into Japanese without 
any linguistic errors caused by the translation is necessary. For further study, it will be interesting to ask a professional translator to translate summaries into Japanese.

Another concern is that we used CV papers only. Papers in other disciplinary areas might have different characteristics. Therefore, using papers in different fields such as humanity or social science will be an important research topic.

Finally, evaluations in both experiments 1 and 2 are highly subjective. In experiment 1 , we asked the subjects to judge which summary was better or not. Each subject could evaluate summaries with their criteria, which is highly subjective. However, we can identify what characteristics the subjects think are necessary for a good summary. One future direction will be interviewing each subject so that we can deeply understand their evaluation criteria. In experiment 2, the subjects were asked to judge whether they would like to read the original paper. However, each subject has their preference on the topic, which could affect the answers. We designed this experiment to realize the users' behavior under the practical scenario of finding relevant papers by reading summaries. A detailed analysis of an individual case is meaningful, including the relationship between subjects preferences and scores in experiment 2. Additionally, though we shuffled the order of summaries for each subject, there is no guarantee that reading different orders by the same subject does not affect the result. Comparison among subjects who read different orders but have similar preferences will be also important to consider the bias.

\section{Conclusions}

We conducted two user tests on a sample of Japanese university students' to gauge their opinions and preferences concerning scientific paper summarization. In experiment 1 , we asked subjects to compare several summaries generated by manual and automatic methods and found unique characteristics of scientific publications which readers regard as important (e.g., structures of research articles and specialized terminology). In addition, whereas automatic machine translation tools such as the Google translate are prone to grammatical and word errors, some subjects, especially low-level and medium-level students, provided positive feedback that translations into their mother tongue (in this paper, Japanese) is better. In experiment 2, we provided subjects with several summaries, and asked them whether they would like to read the original papers after reading the summaries. The experimental results showed that specific components of scientific publications (i.e., the topic and methods) are pivotal for determining subjects' judgments and decisions.

In future work, we will build a summarization system that considers the user's preference. This direction enables efficiently exploring important information in scientific papers for judging which papers to read. We also plan to simplify the paper's terminologies [36] that could create confusion in understanding and improve machine translation systems. This could have important advantages for students without prior knowledge of scientific research and/or languages other than their mother tongue. We believe that the results presented in this paper offer a step toward the acceleration of research in the field of scientific paper summarizations.

Author Contributions: Conceptualization, S.Y. and R.S.; methodology, S.Y. and R.S.; software, S.Y.; validation, S.Y., R.S. and T.F.; formal analysis, S.Y.; investigation, S.Y.; resources, S.Y.; data curation, S.Y.; writing-original draft preparation, S.Y.; writing-review and editing, R.S., T.F., H.K. and S.M.; visualization, S.Y.; supervision, H.K. and S.M.; project administration, H.K. and S.M.; funding acquisition, S.M. All authors have read and agreed to the published version of the manuscript.

Funding: This work was supported by the JST-Mirai Program (JPMJMI19B2) and JSPS KAKENHI (JP19H01129).

Institutional Review Board Statement: Ethical review and approval were waived for this study because any kind of invasion to human subjects was not involved.

Informed Consent Statement: Patient consent was waived because any kind of invasion to human subjects was not involved. 


\section{Data Availability Statement: Not applicable.}

Conflicts of Interest: The authors declare no conflict of interest.

Sample Availability: The data used to support the findings of this study are available from the corresponding author upon request.

\begin{tabular}{|c|c|}
\hline \multicolumn{2}{|c|}{ Abbreviations } \\
\hline \multicolumn{2}{|c|}{ The following abbreviations are used in this manuscript: } \\
\hline $\mathrm{HCI}$ & Human-Computer Interaction \\
\hline $\mathrm{CV}$ & Computer Vision \\
\hline NLP & Natural Language Processing \\
\hline CVPR & the IEEE/CVF Conference on Computer Vision and Pattern Recognition \\
\hline MJ & Manual Japanese \\
\hline $\mathrm{AbE}$ & Abstract English \\
\hline $\mathrm{AuE}$ & Automatic English \\
\hline $\mathrm{AbJ}$ & Abstract Japanese \\
\hline $\mathrm{AuJ}$ & Automatic Japanese \\
\hline
\end{tabular}

\section{References}

1. Bornmann, L.; Mutz, R. Growth rates of modern science: A bibliometric analysis based on the number of publications and cited references. J. Assoc. Inf. Sci. Technol. 2015, 66, 2215-2222. [CrossRef]

2. Jiang, F.K.; Hyland, K. Metadiscursive nouns: Interaction and cohesion in abstract moves. Engl. Specif. Purp. 2017, 46, 1-14. [CrossRef]

3. Elkiss, A.; Shen, S.; Fader, A.; Erkan, G.; States, D.; Radev, D. Blind Men and Elephants: What Do Citation Summaries Tell Us about a Research Article? J. Am. Soc. Inf. Sci. Technol. 2008, 59, 51-62. [CrossRef]

4. Cohan, A.; Goharian, N. Scientific document summarization via citation contextualization and scientific discourse. Int. J. Digit. Libr. 2018, 19, 287-303. [CrossRef]

5. Matsumura, K.; Ogata, M.; Ono, T.; Kato, J.; Sakaguchi, S.; Sakamoto, D.; Sugimoto, M.; Sumi, Y.; Nakamura, H.; Nishida, T.; et al. CHI Benkyokai 2017: Nettowâku Renkei Shita Benkyokai to Sono Shien Shisutemu. [CHI Study Meeting 2017: Networked Study Meeting and Support System.] (In Japanese, Title Translated by the Author of This Article). IPSJ SIG Technical Reports. 2017. Volume 2017-HCI-174, pp. 1-8. Available online: https://ipsj.ixsq.nii.ac.jp/ej/index.php?active_action=repository_view_main_ item_detail\&page_id=13\&block_id=8\&item_id=183069\&item_no=1 (accessed on 9 June 2021).

6. Kataoka, H.; Shirakabe, S.; He, Y.; Ueta, S.; Suzuki, T.; Abe, K.; Kanezaki, A.; Morita, S.; Yabe, T.; Kanehara, Y.; et al. cvpaper. challenge in 2016: Futuristic Computer Vision through 1600 Papers Survey. arXiv 2017, arXiv:1707.06436.

7. Gambhir, M.; Gupta, V. Recent automatic text summarization techniques: a survey. Artif. Intell. Rev. 2017, 47, 1-66. [CrossRef]

8. Neubig, G.; Watanabe, T. Optimization for statistical machine translation: A survey. Comput. Linguist. 2016, 42, 1-54. [CrossRef]

9. $\quad$ Erera, S.; Shmueli-Scheuer, M.; Feigenblat, G.; Nakash, O.P.; Boni, O.; Roitman, H.; Cohen, D.; Weiner, B.; Mass, Y.; Rivlin, O.; et al. A Summarization System for Scientific Documents. In Proceedings of the 2019 Conference on Empirical Methods in Natural Language Processing and the 9th International Joint Conference on Natural Language Processing (EMNLP-IJCNLP): System Demonstrations, Hong Kong, China, 3-7 November 2019; pp. 211-216.

10. Cohan, A.; Goharian, N. Scientific Article Summarization Using Citation-Context and Article's Discourse Structure. In Proceedings of the 2015 Conference on Empirical Methods in Natural Language Processing, Lisbon, Portugal, 17-21 September 2015; pp. 390-400.

11. Kryscinski, W.; Keskar, N.S.; McCann, B.; Xiong, C.; Socher, R. Neural Text Summarization: A Critical Evaluation. In Proceedings of the 2019 Conference on Empirical Methods in Natural Language Processing and the 9th International Joint Conference on Natural Language Processing (EMNLP-IJCNLP), Hong Kong, China, 3-7 November 2019; pp. 540-551.

12. Lin, C.Y. Rouge: A package for automatic evaluation of summaries. In Text Summarization Branches Out; Association for Computational Linguistics: Barcelona, Spain, 2004; pp. 74-81.

13. Yasunaga, M.; Kasai, J.; Zhang, R.; Fabbri, A.R.; Li, I.; Friedman, D.; Radev, D.R. Scisummnet: A large annotated corpus and content-impact models for scientific paper summarization with citation networks. In Proceedings of the AAAI Conference on Artificial Intelligence, Honolulu, HI, USA, 27 January-1 February 2019; pp. 7386-7393.

14. Cohan, A.; Dernoncourt, F.; Kim, D.S.; Bui, T.; Kim, S.; Chang, W.; Goharian, N. A Discourse-Aware Attention Model for Abstractive Summarization of Long Documents. In Proceedings of the NAACL-HLT, New Orleans, LA, USA, 1-6 June 2018; pp. 615-621.

15. Nikolov, N.I.; Pfeiffer, M.; Hahnloser, R.H. Data-driven Summarization of Scientific Articles. In Proceedings of the WOSP 2018 Workshop Proceedings. European Language Resources Association, Paris, France, 5-6 December 2018; p. 2_W24 
16. Collins, E.; Augenstein, I.; Riedel, S. A Supervised Approach to Extractive Summarisation of Scientific Papers. In Proceedings of the 21st Conference on Computational Natural Language Learning (CoNLL 2017), Vancouver, BC, Canada, 3-4 August 2017; pp. 195-205.

17. Suppe, F. The structure of a scientific paper. Philos. Sci. 1998, 65, 381-405. [CrossRef]

18. Lloret, E.; Plaza, L.; Aker, A. The challenging task of summary evaluation: an overview. Lang. Resour. Eval. 2018, 52, 101-148. [CrossRef]

19. Huang, D.; Cui, L.; Yang, S.; Bao, G.; Wang, K.; Xie, J.; Zhang, Y. What Have We Achieved on Text Summarization? In Proceedings of the 2020 Conference on Empirical Methods in Natural Language Processing (EMNLP), Onlin, 16-20 November 2020; pp. 446-469.

20. Hermann, K.M.; Kočiskỳ, T.; Grefenstette, E.; Espeholt, L.; Kay, W.; Suleyman, M.; Blunsom, P. Teaching machines to read and comprehend. Adv. Neural Inf. Process. Syst. 2015, 28, 1693-1701.

21. Ozuru, Y.; Dempsey, K.; McNamara, D.S. Prior knowledge, reading skill, and text cohesion in the comprehension of science texts. Learn. Instr. 2009, 19, 228-242. [CrossRef]

22. Shepherd, M.D.; Van De Sande, C.C. Reading mathematics for understanding-from novice to expert. J. Math. Behav. 2014, 35, 74-86. [CrossRef]

23. August, T.; Card, D.; Hsieh, G.; Smith, N.A.; Reinecke, K. Explain like I am a Scientist: The Linguistic Barriers of Entry to r/science. In Proceedings of the 2020 CHI Conference on Human Factors in Computing Systems, Honolulu, HI, USA, 25-30 April 2020; pp. 1-12.

24. Lee, O.; Buxton, C.A. Integrating science and English proficiency for English language learners. Theory Pract. 2013, 52, 36-42. [CrossRef]

25. Tobin, K.; McRobbie, C.J. Significance of limited English proficiency and cultural capital to the performance in science of Chinese-Australians. J. Res. Sci. Teach. Off. J. Natl. Assoc. Res. Sci. Teach. 1996, 33, 265-282. [CrossRef]

26. Wu, Y.; Schuster, M.; Chen, Z.; Le, Q.V.; Norouzi, M.; Macherey, W.; Krikun, M.; Cao, Y.; Gao, Q.; Macherey, K.; et al. Google's neural machine translation system: Bridging the gap between human and machine translation. arXiv 2016, arXiv:1609.08144.

27. Mihalcea, R.; Tarau, P. Textrank: Bringing order into text. In Proceedings of the 2004 Conference on Empirical Methods in Natural Language Processing, Barcelona, Spain, 25-26 July 2004; pp. 404-411.

28. Barrios, F.; López, F.; Argerich, L.; Wachenchauzer, R. Variations of the Similarity Function of TextRank for Automated Summarization. arXiv 2016, arXiv:1602.03606.

29. Caine, K. Local standards for sample size at CHI. In Proceedings of the 2016 CHI Conference on Human Factors in Computing Systems, San Jose, CA, USA, 7-12 May 2016; pp. 981-992.

30. Scheffé, H. An analysis of variance for paired comparisons. J. Am. Stat. Assoc. 1952, 47, 381-400.

31. Nakaya, S. Scheffé no Ittuihikakuhou no Itihenpou [A Variation of Scheffé's Analysis of Variance for Paired Comparisons]. (In Japanese, Title Translated by the Author of This Article). pp. 1-12. Available online: https://ci.nii.ac.jp/naid/10009334165/ (accessed on 9 June 2021).

32. Cachola, I.; Lo, K.; Cohan, A.; Weld, D.S. TLDR: Extreme Summarization of Scientific Documents. In Proceedings of the 2020 Conference on Empirical Methods in Natural Language Processing: Findings, Onlin , 16-20 November 2020; pp. $4766-4777$.

33. Nenkova, A.; Passonneau, R.J. Evaluating content selection in summarization: The pyramid method. In Proceedings of the Human Language Technology Conference of the North American Chapter of the Association for Computational Linguistics: Hlt-naacl 2004, Boston, MA, USA, 2-7 May 2004; pp. 145-152.

34. Surya, S.; Mishra, A.; Laha, A.; Jain, P.; Sankaranarayanan, K. Unsupervised Neural Text Simplification. In Proceedings of the 57th Annual Meeting of the Association for Computational Linguistics, Florence, Italy, 28 July-2 August 2019; pp. $2058-2068$.

35. Hsu, W.T.; Lin, C.K.; Lee, M.Y.; Min, K.; Tang, J.; Sun, M. A Unified Model for Extractive and Abstractive Summarization using Inconsistency Loss. In Proceedings of the 56th Annual Meeting of the Association for Computational Linguistics (Volume 1: Long Papers), Melbourne, Australia, 15-20 July 2018; pp. 132-141.

36. Kim, Y.S.; Hullman, J.; Burgess, M.; Adar, E. Simplescience: Lexical simplification of scientific terminology. In Proceedings of the 2016 Conference on Empirical Methods in Natural Language Processing, Austin, TX, USA, 1-5 November 2016; pp. $1066-1071$. 